Editorial

\title{
Anemia during Direct-Acting Antiviral Regimens in Kidney Transplant Recipients with Hepatitis C
}

Tatsuo Kanda ${ }^{1,} *$, Shunich Matsuoka ${ }^{1}$, Mitsuhiko Moriyama ${ }^{1}$

1. Division of Gastroenterology and Hepatology, Department of Medicine, Nihon University School of Medicine, 30-1 Oyaguchi-Kamicho, Itabashi-ku, Tokyo 173-8610, Japan; E-Mails:

kanda2t@yahoo.co.jp (TK); matsuoka.shunichi@nihon-u.ac.jp (SM);

moriyama.mitsuhiko@nihon-u.ac.jp (MM)

* Correspondence: Tatsuo Kanda; E-Mail: kanda2t@yahoo.co.jp

Academic Editor(s): Xia Jiang

OBM Hepatology and Gastroenterology

2017, volume 1 , issue 4

doi:10.21926/obm.hg.1704005
Received: October, 18, 2017

Accepted: October, 26, 2017

Published: October, 30, 2017

Saxena et al. [1] reported the safety and efficacy of current direct-acting antiviral (DAA) therapies in liver transplant (LT), kidney transplant (KT) and dual liver kidney (DLK) transplant recipients infected with hepatitis $C$ virus (HCV). Moreover, the addition of ribavirin (RBV) to DAA therapies did not influence rates of sustained virologic response (SVR) or the incidence of graft rejection. In addition, patients taking RBV were more likely to develop anemia (36\%) as a common adverse effect, compared with patients not receiving RBV (3\%) [1]. In a similar vein, Fernandez and colleagues [2] reported Grade 2 or 3 anemia in 33\% of patients receiving RBV-containing regimens versus $15 \%$ of patients receiving no RBV. Our results agree with the results above from both studies. Notably, anemia was one of the serious adverse effects we observed in our KT patients with chronic hepatitis $\mathrm{C}$ during treatment with interferon-free regimens [3].

In KT recipients, anemia depends on several factors: presence of advanced liver fibrosis, renal function, and the duration between KT and the start of antiviral treatment. Previous studies had established that interleukin- 6 and lipopolysaccharide stimulate hepatic expression of hepcidin. Hepcidin subsequently inhibits duodenal absorption of iron [4], aligning with observations that

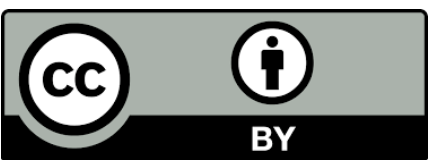

(C) 2017 by the author. This is an open access article distributed under the conditions of the Creative Commons by Attribution License, which permits unrestricted use, distribution, and reproduction in any medium or format, provided the original work is correctly cited. 
iron metabolism appears to be disrupted in patients with advanced liver fibrosis. Alternatively, anemia has also been associated with chronic renal disease, as decreased erythropoietin production was observed to be mediated by renal insufficiency and other factors [4]. Another study has supported this association, demonstrating that approximately one-half of patients were reportedly anemic at six months post-KT, while post-KT anemia was observed in one-third of patients during the first five years [5].

Notably, Saxena et al. reported that their study included $28 \%$ of KT patients with cirrhosis and only $5 \%$ of those had an estimated glomerular filtration rate (eGFR) $\leq 30 \mathrm{~mL} / \mathrm{min}$ [1]. Fernandez et al. [2] also reported a similar percentage (35\%) of KT-patients with cirrhosis, with only $12 \%$ having eGFR $<30 \mathrm{ml} / \mathrm{min}$ and a median interval between KT and the start of antiviral treatment of 147 months. Furthermore, Kogiso et al. [6] reported that $11 \mathrm{KT}$ recipients treated with DAAs exhibited SVR rates of $100 \%$; however, severe anemia was observed in several KT recipients with renal impairment and/or cirrhosis during DAAs treatment for chronic hepatitis $C$ even in the absence of RBV [3].

Comorbidities of HCV infection and chronic kidney disease might present in two ways: HCV infection during dialysis and HCV-associated kidney diseases [7]. Because prevalence of HCV infection in KT recipients is higher than in the general population, careful attention should be paid to administer DAAs without RBV for treatment of KT-recipients with chronic hepatitis C.

\section{Author Contributions}

T.K., S.M. and M.M. searched the literature, discussed the results and wrote the article.

\section{Funding}

T.K. was supported by JSPS KAKENHI Grant Number 17K09404 and AMED.

\section{Competing Interests}

T.K. has received research grants from Chugai Pharmaceutical, MSD, Sysmex, Tsumura and AbbVie.

\section{References}

1. Saxena V, Khungar V, Verna EC, Levitsky J, Brown RS Jr, Hassan MA, et al. Safety and efficacy of current direct-acting antiviral regimens in kidney and liver transplant recipients with hepatitis C: Results from the HCV-TARGET study. Hepatology 2017; 66: 1090-1101.

2. Fernández I, Muñoz-Gómez R, Pascasio JM, Baliellas C, Polanco N, Esforzado N, et al. Efficacy and tolerability of interferon-free antiviral therapy in kidney transplant recipients with chronic hepatitis C. J Hepatol 2017; 66: 718-23.

3. Sasaki R, Kanda T, Yasui S, Haga Y, Nakamura M, Yamato M, et al. Successful Eradication of Hepatitis C Virus by Interferon-Free Regimens in Two Patients with Advanced Liver Fibrosis following Kidney Transplantation. Case Rep Gastroenterol 2016; 10: 248-56.

4. Weiss G, Goodnough LT. Anemia of chronic disease. N Engl J Med 2005; 352: 1011-23.

5. Mix TC, Kazmi W, Khan S, Ruthazer R, Rohrer R, Pereira BJ, et al. Anemia: a continuing problem following kidney transplantation. Am J Transplant 2003; 3: 1426-33. 
6. Kogiso T, Kobayashi M, Yamamoto K, Ikarashi Y, Kodama K, Taniai M, et al. IFN-based and IFNfree direct-acting antiviral drug treatments for acquired hepatitis $C$ virus in post-transplant recipients. OBM Hepatology and Gastroenterology 2017; 1(2). DOI: 10.21926/obm.hg.1702003

7. Omata M, Kanda T, Yu ML, Yokosuka O, Lim SG, Jafri W, et al. APASL consensus statements and management algorithms for hepatitis C virus infection. Hepatol Int 2012; 6: 409-35.

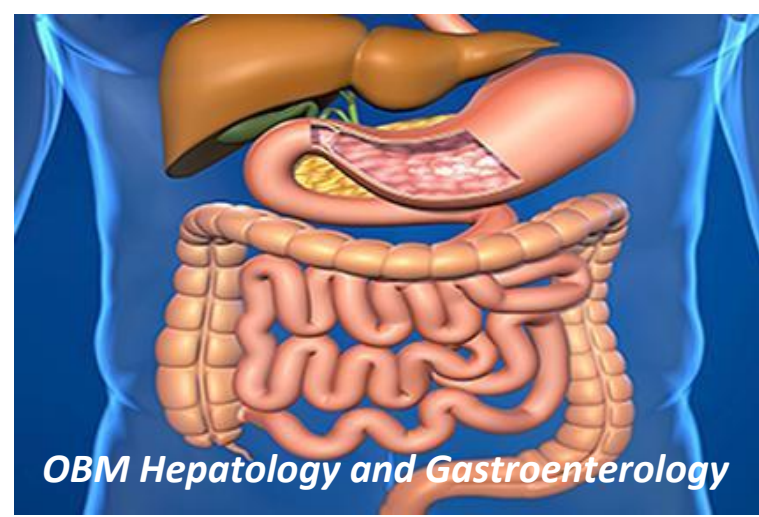

Enjoy OBM Hepatology and Gastroenterology by:

1. Submitting a manuscript

2. Joining in volunteer reviewer bank

3. Joining Editorial Board

4. Guest editing a special issue

For more details, please visit:

http://www.lidsen.com/journals/hg 